\title{
Radiation Necrosis, Pseudoprogression, Pseudoresponse, and Tumor Recurrence: Imaging Challenges for the Evaluation of Treated Gliomas
}

\author{
Anastasia Zikou, ${ }^{1}$ Chrissa Sioka (D, ${ }^{2}$ George A. Alexiou, ${ }^{3}$ Andreas Fotopoulos, ${ }^{2}$ \\ Spyridon Voulgaris, ${ }^{3}$ and Maria I. Argyropoulou ${ }^{1}$ \\ ${ }^{1}$ Department of Radiology, University Hospital of Ioannina, Ioannina, Greece \\ ${ }^{2}$ Department of Nuclear Medicine, University Hospital of Ioannina, Ioannina, Greece \\ ${ }^{3}$ Department of Neurosurgery, University Hospital of Ioannina, Ioannina, Greece \\ Correspondence should be addressed to Chrissa Sioka; csioka@yahoo.com
}

Received 9 July 2018; Accepted 15 October 2018; Published 2 December 2018

Academic Editor: André L. B. de Barros

Copyright ( 2018 Anastasia Zikou et al. This is an open access article distributed under the Creative Commons Attribution License, which permits unrestricted use, distribution, and reproduction in any medium, provided the original work is properly cited.

Glioblastoma (GBM) is the most common primary malignant type of brain neoplasm in adults and carries a dismal prognosis. The current standard of care for GBM is surgical excision followed by radiation therapy (RT) with concurrent and adjuvant temozolomide-based chemotherapy (TMZ) by six additional cycles. In addition, antiangiogenic therapy with an antivascular endothelial growth factor (VEGF) agent has been used for recurrent glioblastoma. Over the last years, new posttreatment entities such as pseudoprogression and pseudoresponse have been recognized, apart from radiation necrosis. This review article focuses on the role of different imaging techniques such as conventional magnetic resonance imaging (MRI), diffusion-weighted imaging (DWI), diffusion tensor imaging (DTI), dynamic contrast enhancement (DCE-MRI) and dynamic susceptibility contrast (DSEMRI) perfusion, magnetic resonance spectroscopy (MRS), and PET/SPECT in differentiation of such treatment-related changes from tumor recurrence.

\section{Introduction}

Glioblastoma (GBM) is the most malignant primary brain tumor in adults with dismal prognosis [1]. Treatment involves gross total excision, when possible, followed by radiotherapy with concurrent and adjuvant temozolomidebased chemotherapy. Nevertheless, nearly all tumors recur. During follow-up, radiotherapy and chemotherapy may produce new lesions that may mimic tumor progression or recurrence on imaging. Accurate detection of glioma recurrence is of paramount importance as it can change the patient's management $[2,3]$. To date, several imaging modalities have been employed for the differentiation of glioma recurrence from treatment-induced changes [2-5]. Herewith, we reviewed the current evidence on the ability of imaging techniques such as MRI, SPECT, and PET for the detection of glioma recurrence/progression.
1.1. Radiation Necrosis. Radiation necrosis (RN) in patients with malignant gliomas is a severe local tissue reaction to radiotherapy. It generally occurs 3-12 months after radiotherapy but can occur up to several years and even decades later $[2,6]$. A complete understanding of the pathophysiology of RN and chemotherapy-induced injury to the central nervous system is useful in understanding and interpreting the conventional and advanced diagnostic imaging findings. The proposed mechanisms by which radiation-associated neurotoxicity may be developed are vascular injury, glial and white matter damage, and the impact on the fibrinolytic enzyme system [7]. In acute radiation-induced injury, transient vasodilatation occurs with variable changes in capillary permeability that sometimes manifest as vasogenic edema. In chronic radiation-induced injury, vascular endothelial damage takes place. Animal studies showed that, in the beginning, there were vascular abnormalities and 
parenchymal changes in the brain followed. The pathological findings are mainly endothelial damage, vascular dilation, and telangiectasia. These have an effect on capillary permeability that produces cytotoxic and vasogenic edema [8]. Thus, vascular damage has a critical role in the development of radiation-induced effects in the brain; however, further research is needed. One different concept is that cytokine release may promote angiogenesis, which is associated with capillary leakage. The main cytokine secreted after irradiation is tumor necrosis factor-alpha (TNF- $\alpha$ ). TNF- $\alpha$ upregulates other cytokines that induce apoptosis of the endothelial cells, astrocyte activation, and blood-brain barrier (BBB) permeability $[9,10]$. The vascular endothelial growth factor (VEGF) induces small vessel permeability and causes cerebral edema. Increased VEGF expression has been reported in the white matter after radiotherapy. VEGF expression has been associated with the magnitude of edema and breakdown of the BBB. Presence of radiation necrosis has been linked to increased VEGF expression [11].

Glial and white matter damage happens after irradiation. Although neurons are relatively insensitive to radiation, it has been shown that oligodendrocytes have higher sensitivity to ionizing radiation and their damage is associated with radiologic evidence of the demyelination that follows [12]. Additionally, there are effects from radiation on the fibrinolytic enzyme system such as the tissue plasminogen and urokinase plasminogen activators, which produce several effects on blood vessels and brain tissue. Sawaya et al. examined irradiated rat cervical spinal cords and proposed the role of plasminogen activators in the pathogenic pathways of radiation damage. An absence of tissue plasminogen activator and an increase in urokinase plasminogen activator leading to cytotoxic edema and tissue necrosis have been reported [13]. Based on that, efforts have been made in the development of radiosensitizers, agents that can be administered prior or concurrent with radiotherapy in order to augment the sensitivity of the tumor, while not affecting normal brain tissue. Thus, radiation dose reduction can be made [14]. In glioblastoma treatment, the chemotherapeutic agent temozolomide is an example of a radiosensitizer. When standard radiotherapy was compared with identical radiotherapy with concomitant temozolomide, an increase in the median survival was found (12.1 vs 14.6 months) [15]. A better understanding of the molecular pathways involved in tumor's radioresistance may reveal new potential therapeutic targets $[16,17]$.

Radiation necrosis is characterized histopathologically by fibrinoid necrosis of blood vessel walls, with adjacent perivascular parenchymal coagulative necrosis. Collections of abnormally dilated and thin-walled telangiectasias can be also observed. Hyalinization caused vessel wall thickening and is a late finding. Focal and diffuse demyelination constitutes the white matter changes observed.

1.2. Pseudoprogression. After completion of RT, with or without concomitant TMZ, patients with high-grade brain tumors can present with new lesions or with an increase in contrast-enhancing previous lesions and perilesional edema. Pseudoprogression has been reported to occur predominantly (in almost $60 \%$ of cases) within the first 3 months after completing treatment, but it may occur later after treatment with iomustine and temozolomide $[18,19]$. The $\mathrm{O}^{6}$ methylguanine-DNA methyltransferase (MGMT) methylation tumor's status has been associated with pseudoprogression occurrence and 2/3 of MGMT methylated tumors exhibit pseudoprogression, $11 \%$ early progression, and $25 \%$ stable disease. Brades et al. proposed that tumors with methylation of the MGMT promoter, due to greater effect of the combination of temozolomide and radiotherapy to residual tumor, produce a temporary worsening of imaging characteristics which are characterized as pseudoprogression [20]. Hegi et al. showed that patients with methylated MGMT promoter status had better median overall survival (43.6 months vs 16.8 months) [21]. On the other hand, a $60 \%$ probability of early true tumor progression in unmethylated MGMT promoter tumors was reported [21]. The exact pathophysiological features of pseudoprogression and the associated molecular changes require further research. Pseudoprogression may constitute an overresponse to effective therapy and is associated with damage to the endothelium, BBB disruption, and oligodendroglial injury $[18,22]$.

It is important for pathologists to be aware of concerns regarding the accurate diagnosis of this phenomenon. Heterogeneity might be the hallmark when analyzing biopsy samples in these cases. Even within pathologists, there might be variability in the interpretation of findings [23].

1.3. Pseudoresponse. Recently, anti-VEGF agents have been utilized for high-grade glioma treatment in several trials. Anti-VEGF agents produce "normalization" of the bloodbrain barrier, sometimes within hours. On imaging, there is a reduction in the degree of enhancement by the tumor and a decrease in the surrounding edema on fluid-attenuated inversion recovery (FLAIR). Such an imaging appearance, which imitates a favorable treatment response, is termed "pseudoresponse" because this is due to alterations in vascular permeability instead of tumor response to treatment. So, this radiologic response should be interpreted with caution. Furthermore, studies have shown that antiangiogenic agents, although they cause great imaging changes of the tumor's appearance, overall survival has only a modest increase. Additionally, there might be a rebound effect later with the presence of enhancement and edema $[24,25]$.

\section{Diagnostic Imaging Modalities}

2.1. Conventional and Advanced MR Imaging Techniques. Imaging plays a key role in assessment of response to various treatment regimens for high-grade gliomas. T1-weighted contrast-enhanced MR imaging should be used within the 2 days after surgery in order to assess extent of resection and no later than 72 hours after operation. Moreover, increased contrast enhancement detected by MR imaging just after or during treatment can be produced by several causes such postoperative changes, microischemic lesions, and treatment-associated inflammation. For years, a set of 
guidelines by Macdonald et al. (Macdonald criteria) were used to evaluate the tumor's treatment response. Based on that, four response categories were identified: complete response, partial response, progressive disease, and stable disease [26]. Limitation of this scheme was that the presence of post-treatment contrast enhancement might not be associated with tumor activity but with BBB disruption. Furthermore, based on the latest RANO criteria, the nonenhancing tumor's component should also be evaluated for decision-making [27]. The most common imaging patterns of radiation necrosis in conventional MRI include a single lesion arising at the resection cavity which can be interpreted for recurrent tumor; a necrosis far from the primary tumor site may mimic multifocal glioma; the "Swiss cheese" pattern can be visualized as diffuse enhancements at the margins between the cortex and white matter.

Regarding the advanced MR techniques, a recent metaanalysis that included 35 studies of all diagnostic MRI techniques in high-grade glioma patients after treatment showed that advanced MRI techniques had higher diagnostic accuracy than conventional MRI for the detection of tumor progression. MR spectroscopy showed the highest diagnostic accuracy followed by perfusion MRI. The sensitivity and specificity of MRS were $91 \%$ and $95 \%$, respectively [28]. In a retrospective study of 15 patients with lesions suspicious for glioma progression, a cutoff value of 1.30 in ADC ratio, 2.10 for $\mathrm{rCBV}$ ratio, 1.29 for $\mathrm{Cho} / \mathrm{Cr}$ ratio, and 1.06 for Cho/NAA ratio had a diagnostic accuracy of $86.7 \%, 86.7 \%$, and $84.6 \%$, respectively. When an analysis of a combination of parameters was performed, the diagnostic accuracy reached $93.3 \%$ [29]. Patel et al. also performed a systematic review and metaanalysis to evaluate whether DSC and DCE metrics could differentiate recurrent glioma from posttreatment changes, including both pseudoprogression and radiation necrosis. The meta-analysis included 28 studies, 13 of which evaluated pseudoprogression. The results showed that, for DSC, the pooled sensitivity was $90 \%$ and the specificity was $88 \%$. For DCE, the pooled sensitivity was $89 \%$ and the specificity was $85 \%$ [30]. It is of notice that a recent survey on glioma imaging practices of the members of the European Society of Neuroradiology from 220 institutions showed that perfusion MRI is widely used (85.5\%). Spectroscopy is used mainly for specific indications [31].

2.2. PET. In a study of fifty consecutive patients, hybrid ${ }^{11} \mathrm{C}$ methyl-L-methionine ( ${ }^{11} \mathrm{C}$-MET) PET/MRI showed significant higher accuracy than MRI (96\% vs $82 \%$ ) and higher to ${ }^{11}$ C-MET PET (96\% vs $88 \%$ ) to differentiate treatmentrelated changes from true progression in recurrent glioma, based on RANO criteria [32]. A maximum tumor-tobrain ratio (TBR) of 1.83 and mean TBR of 1.5 were found as optimal cutoff values for discriminating between these two entities [32]. ${ }^{11} \mathrm{C}-\mathrm{METPET} / \mathrm{CT}$ differentiated recurrence from no recurrence with a cutoff $\max \mathrm{T} / \mathrm{N}$ ratio of 1.9 , with a sensitivity of $94.7 \%$ and a specificity of $88.89 \%$ [33]. A limitation of ${ }^{11} \mathrm{C}$-MET is the need for an on-site cyclotron due to short half life of ${ }^{11} \mathrm{C}$, making widespread use of this tracer difficult [34].
O-(2-(18)F-fluoroethyl)-L-tyrosine $\left({ }^{18} \mathrm{~F}-\mathrm{FET}\right)$ is an artificial amino acid and a promising tracer for the detection of recurrent glioma. ${ }^{18} \mathrm{~F}$-fluorine tracers do not require an onsite cyclotron. In a study of 22 glioblastoma patients that presented with increased enhancement of lesions or new contrast-enhancing lesions within the first 3 months after completion of radiochemotherapy, ${ }^{18}$ F-FET PET could identify pseudoprogression with $96 \%$ accuracy. Moreover, survival analysis showed that a max $\mathrm{T} / \mathrm{N}$ ratio lower than 2.3 predicted a significantly longer overall survival [35]. Compared to conventional MRI, ${ }^{18} \mathrm{~F}$-FET PET had higher accuracy (93\% vs $86 \%$, respectively) [30]. Using dynamic and static ${ }^{18}$ F-FET uptake parameters, late pseudoprogression could be differentiated from glioblastoma recurrence, with a cutoff value of $\max \mathrm{T} / \mathrm{N}$ of 1.9 with $84 \%$ sensitivity and $86 \%$ specificity. Concerning clinical decisionmaking, all cases with $\max \mathrm{T} / \mathrm{N}$ ratio greater than 2.4 were glioblastoma recurrence and all cases with $\max \mathrm{T} / \mathrm{N}$ ratio lower than 1 were pseudoprogression [36]. In a welldesigned prospective study that compared ${ }^{11} \mathrm{C}-\mathrm{MET}$ PET to ${ }^{18} \mathrm{~F}$-FET, both tracers showed the same sensitivity (91\%) and specificity (100\%) for differentiating tumor tissue from treatment-related changes [38].

The most widely available PET tracer is the 2-deoxy-2(18F) fluoro-D-glucose $\left({ }^{18} \mathrm{FDG}\right)$, whose function is based on glycolytic metabolism. Thus, lesions with high glucose metabolism show increased uptake. One major disadvantage of ${ }^{18} \mathrm{~F}$-FDG is the high uptake in the normal brain; thus, a lesion is not readily identifiable at all times [3]. The accuracy of ${ }^{18}$ F-FDG PET in the detection of recurrent tumor has been questioned, and reported sensitivities and specificities vary widely [39]. However, a recent metaanalysis showed that ${ }^{11} \mathrm{C}$-MET does not have noticeable advantage over ${ }^{18}$ F-FDG [40]. The use of ${ }^{11} \mathrm{C}$-choline PET/CT demonstrated higher sensitivity and specificity compared with ${ }^{18} \mathrm{~F}-\mathrm{FDG} \mathrm{PET} / \mathrm{CT}$ for distinguishing recurrent brain tumor from radionecrosis [41]. Similarly, another promising PET radiopharmaceutical represents the 3,4-dihydroxy-6-[(18)F] fluoro-phenylalanine $\left({ }^{18} \mathrm{~F}\right.$ FDOPA), which may be highly sensitive and specific for detection of recurrence in glioma patients [42]. Jena et al. studied 35 glioma-treated patients harbouring 41 enhancing lesions with ${ }^{18} \mathrm{~F}-\mathrm{FDG}$ PET/MRI imaging. The accuracy of perfusion MRI (rCBV) for detecting glioma recurrence was $77.5 \%, 78 \%$ for $\mathrm{ADC}$ mean, $90.9 \%$ for Cho/Cr, $87.8 \%$ for $\max \mathrm{T} / \mathrm{N}$, and $87.8 \%$ for mean $\mathrm{T} / \mathrm{N}$. On multivariate ROC analysis, the maximum area under the curve (AUC) of $0.935 \pm 0.046$ was achieved when ADC mean, Cho/Cr, and TBRmean were combined [43]. Table 1 summarizes representative PET studies for the detection of recurrent glioma [32-34, 36, 37, 43-47].

Other PET radiopharmaceuticals that are under investigation to differentiate between progression from pseudoprogression or tumor recurrence include ${ }^{18} \mathrm{~F}$-fluor omisonidazole (FMISO) PET/MR [48], 4-borono-2-[(18) F]-fluoro-phenylalanine ( ${ }^{18} \mathrm{~F}$-FBPA) PET [49], [(18)F]fluoromethyl-dimethyl-2-hydroxyethylammonium $\left({ }^{18} \mathrm{~F}\right.$-flu oromethylcholine) PET [50], and $3^{\prime}$-deoxy- $3^{\prime}-(18) \mathrm{F}-$ fluorothymidine (FLT) PET [51]. 
TABle 1: Cutoff values of PET tracers for the detection of glioma recurrence.

\begin{tabular}{|c|c|c|c|c|c|c|}
\hline Study & Modality & Tracer & Parameter & Cutoff & Sensitivity (\%) & Specificity (\%) \\
\hline Deuschl et al. [32] & PET/MRI & ${ }^{11} \mathrm{C}$-methionine & $\begin{array}{c}\text { Max T/N } \\
\text { Mean T/N }\end{array}$ & $\begin{array}{l}1.8 \\
1.3\end{array}$ & 97.14 & 93.33 \\
\hline Tripathi et al. [33] & $\mathrm{PET} / \mathrm{CT}$ & ${ }^{11} \mathrm{C}$-methionine & $\operatorname{Max} \mathrm{T} / \mathrm{N}$ & 1.9 & 95 & 89 \\
\hline Terakawa et al. [34] & PET & ${ }^{11} \mathrm{C}$-methionine & Mean $\mathrm{T} / \mathrm{N}$ & 1.58 & 75 & 75 \\
\hline Herholz et al. [44] & PET & ${ }^{11} \mathrm{C}$-methionine & $\mathrm{T} / \mathrm{N}$ & 1.47 & 76 & 87 \\
\hline Garcia et al. [45] & PET & ${ }^{11} \mathrm{C}$-methionine & $\mathrm{T} / \mathrm{N}$ & 2.35 & 90.5 & 100 \\
\hline Takenaka et al. [46] & PET & ${ }^{11} \mathrm{C}$-methionine & $\mathrm{T} / \mathrm{N}$ & 2.51 & 91.2 & 81.4 \\
\hline Galldinks et al. [36] & PET & ${ }^{18} \mathrm{~F}-\mathrm{FET}$ & $\operatorname{Max} \mathrm{T} / \mathrm{N}$ & 2.3 & 100 & 91 \\
\hline Kebir et al. [37] & PET & ${ }^{18} \mathrm{~F}-\mathrm{FET}$ & $\operatorname{Max} \mathrm{T} / \mathrm{N}$ & 1.9 & 84 & 86 \\
\hline \multirow{2}{*}{ Jena et al. [43] } & \multirow{2}{*}{ PET/MRI } & \multirow{2}{*}{${ }^{18} \mathrm{~F}-\mathrm{FDG}$} & $\operatorname{Max} \mathrm{T} / \mathrm{N}$ & 1.579 & 93.3 & 72.7 \\
\hline & & & Mean $\mathrm{T} / \mathrm{N}$ & 1.179 & 90 & 81.8 \\
\hline \multirow{2}{*}{ Enslow et al. [47] } & \multirow{2}{*}{ PET } & \multirow{2}{*}{${ }^{18} \mathrm{~F}-\mathrm{FDG}$} & $\mathrm{T} / \mathrm{N}$ & 1.83 & 100 & 75 \\
\hline & & & Max T/N & 6.2 & 90.9 & 75 \\
\hline
\end{tabular}

2.3. SPECT. Compared to PET, SPECT is widely available and of lower cost; however, it has lower spatial resolution. Several SPECT tracers have been evaluated mainly for the differentiation of glioma recurrence from radiation necrosis. ${ }^{201}$ Thallium $\left({ }^{201} \mathrm{Tl}\right.$ ) was one of the first SPECT tracers studied [52]. Given that there is no $\mathrm{Tl}$ uptake in the healthy brain, tumor recurrence can be readily identified. The sensitivity of ${ }^{201} \mathrm{Tl}$ SPECT for the detection of recurrent glioma ranged from 0.43 to 1.00 , and the specificity ranged from 0.25 to 1.00 [52]. Technetium-99m-labeled compounds are superior to ${ }^{201} \mathrm{Tl}$ given the $140 \mathrm{keV} \gamma$-ray energy and high photon flux that correspond to higher spatial resolution and less radiation burden to the patient [53]. ${ }^{99 \mathrm{~m}} \mathrm{Tc}$-sestamibi and ${ }^{99 \mathrm{~m}} \mathrm{Tc}$ tetrofosmin have been found suitable for the detection of recurrent tumors. Both tracers have no uptake in the healthy brain except areas with absence of BBB such as choroid plexus. A cutoff value of lesion-to-tumoral uptake ratio around 4 has been reported for the differentiation of recurrent tumor from radiation necrosis [53-55].

It is of interest that ${ }^{99 \mathrm{~m}} \mathrm{Tc}$-tetrofosmin SPECT was found to have the same accuracy with perfusion MRI to detect recurrent tumor following glioma treatment [55]. ${ }^{99 \mathrm{~m}} \mathrm{Tc}$-methionine andiodine-123-a-methyl tyrosine $\left({ }^{123} \mathrm{I}-\right.$ IMT) has been also evaluated for the detection of radiation necrosis [56]. Amino acid-based tracers have an uptake in the healthy brain, contrary to the previous reported tracers. Recently, in a study of 44 patients with suspected glioma recurrence, ${ }^{99}$ Tc-methionine SPECT/CT had similar diagnostic values with FDG PET/CT and higher than contrast-enhanced MRI for the detection of glioma recurrence. The sensitivity and specificity of ${ }^{99 \mathrm{~m}} \mathrm{Tc}$ methionine, FDG PET/CT, and contrast-enhanced MRI was $75.9 \%$ and $90 \%, 82.8 \%$ and $80 \%$, and $87.1 \%$ and $30 \%$, respectively [57]. In a meta-analysis that evaluated the diagnostic ability of SPECT in differentiating glioma recurrence from radiation necrosis, the pooled sensitivity was $89 \%$ and the specificity $88 \%$ [58]. Other promising tracers for SPECT to differentiate tumor recurrence from radiation necrosis include pentavalent technetium-99m dimercaptosuccinic acid (Tc-99m (V) DMSA) [59], bis-methionineDTPA (Tc MDM) [60], and 99m-technetium glucoheptonate ( $\left.{ }^{99 \mathrm{~m}} \mathrm{Tc}-\mathrm{GHA}\right)$ [61].

\section{Conclusions}

Pseudoprogression is a major concern in glioma-treated patients and has a reported incidence between 10 and $30 \%$. It usually appears several weeks up to approximately 4 months after radiotherapy. Thus, patients are not eligible to enter clinical trials within 4 months after treatment, because of the risk of unreliable results. Contrary to radiation necrosis, the pathophysiology of pseudoprogression remains elusive and further studies are needed. To date, no single technique provides a reliable detection of glioma recurrence. An imaging modality that could reliably detect tumor progression would be of paramount importance. One major limitation in all previously reported studies is the absence of histopathological confirmation of the final diagnosis in all cases. This might be due to lesion's location in an eloquent brain region or patient denial for biopsy or further surgery. Given all the above, the main focus of future studies should be on the discrimination of recurrence from no recurrence. Well-designed future studies combining several imaging modalities should be performed.

\section{Conflicts of Interest}

Dr. Fotopoulos and Dr. Alexiou took part in the GLIOMARK Project, which received funding from the European Union's Horizon 2020 Research and Innovation, SME Instrument Phase II Programme (GA No. 673737).

\section{References}

[1] Y. Liu, S. Shete, C. J. Etzel et al., "Polymorphisms of LIG4, BTBD2, HMGA2, and RTEL1 genes involved in the doublestrand break repair pathway predict glioblastoma survival," Journal of Clinical Oncology, vol. 28, no. 14, pp. 2467-2474, 2010.

[2] P. Giglio and M. R. Gilbert, "Cerebral radiation necrosis," Neurologist, vol. 9, no. 4, pp. 180-188, 2003.

[3] G. A. Alexiou, S. Tsiouris, A. P. Kyritsis, S. Voulgaris, M. I. Argyropoulou, and A. D. Fotopoulos, "Glioma recurrence versus radiation necrosis: accuracy of current imaging modalities," Journal of Neuro-Oncology, vol. 95, no. 1, pp. 1-11, 2009. 
[4] S. C. Thust, M. J. van den Bent, and M. Smits, "Pseudoprogression of brain tumors," Journal of Magnetic Resonance Imaging, vol. 48, no. 3, pp. 571-589, 2018.

[5] N. Galldiks, M. Kocher, and K. J. Langen, "Pseudoprogression after glioma therapy: an update," Expert Review of Neurotherapeutics, vol. 17, no. 11, pp. 1109-1115, 2017.

[6] O. Kargiotis, A. Geka, J. S. Rao, and A. P. Kyritsis, "Effects of irradiation on tumor cell survival, invasion and angiogenesis," Journal of Neuro-Oncology, vol. 100, no. 3, pp. 323-338, 2010.

[7] A. J. Kumar, N. E. Leeds, G. N. Fuller et al., "Malignant gliomas: MR imaging spectrum of radiation therapy- and chemotherapy-induced necrosis of the brain after treatment," Radiology, vol. 217, no. 2, pp. 377-384, 2000.

[8] J. R. Fike and C. E. Cann, "Contrast medium accumulation and washout in canine brain tumors and irradiated normal brain: a CT study of kinetics," Radiology, vol. 151, no. 1, pp. 115-120, 1984.

[9] R. Ansari, M. W. Gaber, B. Wang, C. B. Pattillo, C. Miyamoto, and M. F. Kiani, "Anti-TNFA (TNF- $\alpha$ ) treatment abrogates radiation-induced changes in vascular density and tissue oxygenation," Radiation Research, vol. 167, no. 1, pp. 80-86, 2007.

[10] C. M. Wilson, M. W. Gaber, O. M. Sabek, J. A. Zawaski, and T. E. Merchant, "Radiation-induced astrogliosis and bloodbrain barrier damage can be abrogated using anti-TNF treatment," International Journal of Radiation Oncology * Biology * Physics, vol. 74, no. 3, pp. 934-941, 2009.

[11] N. van Bruggen, H. Thibodeaux, J. T. Palmer et al., "VEGF antagonism reduces edema formation and tissue damage after ischemia/reperfusion injury in the mouse brain," Journal of Clinical Investigation, vol. 104, no. 11, pp. 1613-1620, 1999.

[12] E. Barbarese and C. Barry, "Radiation sensitivity of glial cells in primary culture," Journal of the Neurological Sciences, vol. 91, no. 1-2, pp. 97-107, 1989.

[13] R. Sawaya, A. Rayford, S. Kono et al., "Plasminogen activator inhibitor-1 in the pathogenesis of delayed radiation damage in rat spinal cord in vivo," Journal of Neurosurgery, vol. 81, no. 3, pp. 381-387, 1994.

[14] K. Kelley, J. Knisely, M. Symons, and R. Ruggieri, "Radioresistance of brain tumors," Cancers, vol. 8, no. 4, p. 42, 2016.

[15] R. Stupp, M. E. Hegi, W. P. Mason et al., "Effects of radiotherapy with concomitant and adjuvant temozolomide versus radiotherapy alone on survival in glioblastoma in a randomised phase III study: 5-year analysis of the EORTC-NCIC trial," The Lancet Oncology, vol. 10, no. 5, pp. 459-466, 2009.

[16] M. Zhang, S. Kleber, M. Röhrich et al., "Blockade of TGF- $\beta$ signaling by the TGF $\beta$ R-I kinase inhibitor LY2109761 enhances radiation response and prolongs survival in glioblastoma," Cancer Research, vol. 71, no. 23, pp. 7155-7167, 2011.

[17] G. A. Alexiou, K. I. Tsamis, E. Vartholomatos et al., "Combination treatment of TRAIL, DFMO and radiation for malignant glioma cells," Journal of Neuro-Oncology, vol. 123, no. 2, pp. 217-224, 2015.

[18] D. Brandsma, L. Stalpers, W. Taal, P. Sminia, and M. J. van den Bent, "Clinical features, mechanisms, and management of pseudoprogression in malignant gliomas," The Lancet Oncology, vol. 9, no. 5, pp. 453-461, 2008.

[19] W. Taal, D. Brandsma, H. G. de Bruin et al., "Incidence of early pseudo-progression in a cohort of malignant glioma patients treated with chemo irradiation with temozolomide," Cancer, vol. 113, no. 2, pp. 405-410, 2008.

[20] A. A. Brandes, A. Tosoni, F. Spagnolli et al., "Disease progression or pseudoprogression after concomitant radiochemotherapy treatment: pitfalls in neurooncology," Neuro-Oncology, vol. 10, no. 3, pp. 361-367, 2008.

[21] M. E. Hegi, A. C. Diserens, T. Gorlia et al., "MGMT gene silencing and benefit from temozolomide in glioblastoma," New England Journal of Medicine, vol. 352, no. 10, pp. 997-1003, 2005.

[22] E. Franceschi, A. Tosoni, E. Pozzati, and A. A. Brandes, "Association between response to primary treatments and MGMT status in glioblastoma," Expert Review of Anticancer Therapy, vol. 8, no. 11, pp. 1781-1786, 2008.

[23] L. C. Hygino da Cruz Jr., I. Rodriguez, R. C. Domingues, E. L. Gasparetto, and A. G. Sorensen, "Pseudoprogression and pseudoresponse: imaging challenges in the assessment of posttreatment glioma," American Journal of Neuroradiology, vol. 32, no. 11, pp. 1978-1985, 2011.

[24] J. L. Clarke and S. Chang, "Pseudoprogression and pseudoresponse: challenges in brain tumor imaging," Current Neurology and Neuroscience Reports, vol. 9, no. 3, pp. 241-246, 2009.

[25] T. N. Kreisl, L. Kim, K. Moore et al., "Phase II trial of singleagent bevacizumab followed by bevacizumab plus irinotecan at tumor progression in recurrent glioblastoma," Journal of Clinical Oncology, vol. 27, no. 5, pp. 740-745, 2009.

[26] D. R. Macdonald, T. L. Cascino, S. C. Schold Jr., and J. G. Cairncross, "Response criteria for phase II studies of supratentorial malignant glioma," Journal of Clinical Oncology, vol. 8, no. 7, pp. 1277-1280, 1990.

[27] P. Y. Wen, D. R. Macdonald, D. A. Reardon et al., "Updated response assessment criteria for high-grade gliomas: Response Assessment in Neuro-Oncology Working Group," Journal of Clinical Oncology, vol. 28, no. 11, pp. 1963-1972, 2010.

[28] B. R. J. Van Dijken, P. J. Van Laar, G. A. Holtman, and A. Van Der Hoorn, "Diagnostic accuracy of magnetic resonance imaging techniques for treatment response evaluation in patients with high-grade glioma, a systematic review and meta-analysis," European Radiology, vol. 27, no. 10, pp. 4129-4144, 2017.

[29] E. Matsusue, J. R. Fink, J. K. Rockhill, T. Ogawa, and K. R. Maravilla, "Distinction between glioma progression and post-radiation change by combined physiologic MR imaging," Neuroradiology, vol. 52, no. 4, pp. 297-306, 2010.

[30] P. Patel, H. Baradaran, D. Delgado et al., "MR perfusionweighted imaging in the evaluation of high-grade gliomas after treatment: a systematic review and meta-analysis," Neuro-Oncology, vol. 19, no. 1, pp. 118-127, 2017.

[31] S. C. Thust, S. Heiland, A. Falini et al., "Glioma imaging in Europe: a survey of 220 centres and recommendations for best clinical practice," European Radiology, vol. 28, no. 8, pp. 3306-3317, 2018.

[32] C. Deuschl, J. Kirchner, T. D. Poeppel et al., "11C-MET PET/ MRI for detection of recurrent glioma," European Journal of Nuclear Medicine and Molecular Imaging, vol. 45, no. 4, pp. 593-601, 2018.

[33] M. Tripathi, R. Sharma, R. Varshney et al., "Comparison of F-18 FDG and C-11 methionine PET/CT for the evaluation of recurrent primary brain tumors," Clinical Nuclear Medicine, vol. 37, no. 2, pp. 158-163, 2012.

[34] Y. Terakawa, N. Tsuyuguchi, Y. Iwai et al., "Diagnostic accuracy of 11C-methionine PET for differentiation of recurrent brain tumors from radiation necrosis after radiotherapy," Journal of Nuclear Medicine, vol. 49, no. 5, pp. 694-699, 2008.

[35] N. Galldiks, V. Dunkl, G. Stoffels et al., "Diagnosis of pseudoprogression in patients with glioblastoma using $\mathrm{O}-\left(2-\left[{ }^{18} \mathrm{~F}\right]\right.$ fluoroethyl)-L-tyrosine PET," European Journal of Nuclear Medicine and Molecular Imaging, vol. 42, no. 5, pp. 685-695, 2015. 
[36] N. Galldiks, G. Stoffels, C. Filss et al., "The use of dynamic $\mathrm{O}-\left(2{ }^{18} \mathrm{~F}\right.$-fluoroethyl $)-1-$ tyrosine PET in the diagnosis of patients with progressive and recurrent glioma," Neuro-Oncology, vol. 17, no. 9, pp. 1293-1300, 2015.

[37] S. Kebir, R. Fimmers, N. Galldiks et al., "Late pseudoprogression in glioblastoma: diagnostic value of dynamic O-(2$\left[{ }^{18} \mathrm{~F}\right]$ fluoroethyl)-L-Tyrosine PET," Clinical Cancer Research, vol. 22, no. 9, pp. 2190-2196, 2016.

[38] A. L. Grosu, S. T. Astner, E. Riedel et al., "An interindividual comparison of O- $\left(2-\left[{ }^{18} \mathrm{~F}\right]\right.$ fluoroethyl)-L-tyrosine (FET)- and L-[methyl-11C]methionine (MET)-PET in patients with brain gliomas and metastases," International Journal of Radiation Oncology * Biology * Physics, vol. 81, no. 4, pp. 1049-1058, 2011.

[39] P. E. Ricci, J. P. Karis, J. E. Heiserman et al., "Differentiating recurrent tumor from radiation necrosis: time for reevaluation of positron emission tomography?," American Journal of Neuroradiology, vol. 19, no. 3, pp. 407-413, 1998.

[40] X. Wang, X. Hu, P. Xie, W. Li, X. Li, and L. Ma, "Comparison of magnetic resonance spectroscopy and positron emission tomography in detection of tumor recurrence in posttreatment of glioma: a diagnostic meta-analysis," AsiaPacific Journal of Clinical Oncology, vol. 11, no. 2, pp. 97-105, 2015.

[41] H. Tan, L. Chen, Y. Guan, and X. Lin, "Comparison of MRI, F-18 FDG, and 11C-choline PET/CT for their potentials in differentiating brain tumor recurrence from brain tumor necrosis following radiotherapy," Clinical Nuclear Medicine, vol. 36, no. 11, pp. 978-981, 2011.

[42] S. Karunanithi, P. Sharma, A. Kumar et al., "18F-FDOPA PET/CT for detection of recurrence in patients with glioma: prospective comparison with 18F-FDG PET/CT," European Journal of Nuclear Medicine and Molecular Imaging, vol. 40, no. 7, pp. 1025-1035, 2013.

[43] A. Jena, S. Taneja, A. Jha et al., "Multiparametric evaluation in differentiating glioma recurrence from treatment-induced necrosis using simultaneous (18)F-FDG-PET/MRI: a singleinstitution retrospective study," American Journal of Neuroradiology, vol. 38, no. 5, pp. 899-907, 2017.

[44] K. Herholz, T. Hölzer, B. Bauer et al., "11C-methionine PET for differential diagnosis of low-grade gliomas," Neurology, vol. 50, no. 5, pp. 1316-1322, 1998.

[45] J. R. Garcia, M. Cozar, M. Baquero et al., "The value of (11)Cmethionine PET in the early differentiation between tumour recurrence and radionecrosis in patients treated for a highgrade glioma and indeterminate MRI," Revista Española de Medicina Nuclear e Imagen Molecular, vol. 36, no. 2, pp. 85-90, 2017.

[46] S. Takenaka, Y. Asano, J. Shinoda et al., "Comparison of (11) C-methionine, (11)C-choline, and (18)F-fluorodeoxyglucosePETfor distinguishing glioma recurrence from radiation necrosis," Neurologia Medico-Chirurgica, vol. 54, no. 4, pp. 280-289, 2014.

[47] M. S. Enslow, L. V. Zollinger, K. A. Morton et al., "Comparison of $18 \mathrm{~F}$-fluorodeoxyglucose and 18F-fluorothymidine PET in differentiating radiation necrosis from recurrent glioma," Clinical Nuclear Medicine, vol. 37, no. 9, pp. 854-861, 2012.

[48] R. F. BarajasK. A. Krohn et al., “Glioma FMISO PET/MR imaging concurrent with antiangiogenic therapy: molecular imaging as a clinical tool in the burgeoning era of personalized medicine," Biomedicines, vol. 4, no. 4, p. 24, 2016.
[49] R. Beshr, K. Isohashi, T. Watabe et al., "Preliminary feasibility study on differential diagnosis between radiation-induced cerebral necrosis and recurrent brain tumor by means of [(18)F]fluoro-borono-phenylalanine PET/CT," Annals of Nuclear Medicine, pp. 1-7, 2018.

[50] J. Bolcaen, B. Descamps, K. Deblaere et al., “(18)Ffluoromethylcholine (FCho), (18)F-fluoroethyltyrosine (FET), and (18)F-fluorodeoxyglucose (FDG) for the discrimination between high-grade glioma and radiation necrosis in rats: a PET study," Nuclear Medicine and Biology, vol. 42, no. 1, pp. 38-45, 2015.

[51] C. G. Brahm, M. W. den Hollander, R. H. Enting et al., "Serial FLT PET imaging to discriminate between true progression and pseudoprogression in patients with newly diagnosed glioblastoma: a long-term follow-up study," European Journal of Nuclear Medicine and Molecular Imaging, vol. 45, no. 13, pp. 2404-2412, 2018.

[52] M. J. Vos, B. N. Tony, O. S. Hoekstra, T. J. Postma, J. J. Heimans, and L. Hooft, "Systematic review of the diagnostic accuracy of $201 \mathrm{Tl}$ single photon emission computed tomography in the detection of recurrent glioma," Nuclear Medicine Communications, vol. 28, no. 6, pp. 431-439, 2007.

[53] G. A. Alexiou, A. D. Fotopoulos, A. Papadopoulos, A. P. Kyritsis, K. S. Polyzoidis, and S. Tsiouris, "Evaluation of brain tumor recurrence by $(99 \mathrm{~m}) \mathrm{Tc}$-tetrofosmin SPECT: a prospective pilot study," Annals of Nuclear Medicine, vol. 21, no. 5, pp. 293-298, 2007.

[54] F. P. Le Jeune, F. Dubois, S. Blond, and M. Steinling, "Sestamibi technetium-99m brain single-photon emission computed tomography to identify recurrent glioma in adults: 201 studies," Journal of Neuro-Oncology, vol. 77, no. 2, pp. 177-183, 2006.

[55] G. A. Alexiou, A. Zikou, S. Tsiouris et al., "Comparison of diffusion tensor, dynamic susceptibility contrast MRI and $(99 \mathrm{~m}) \mathrm{Tc}-$ Tetrofosmin brain SPECT for the detection of recurrent high-grade glioma," Magnetic Resonance Imaging, vol. 32, no. 7, pp. 854-859, 2014.

[56] T. Kuwert, B. Woesler, C. Morgenroth et al., "Diagnosis of recurrent glioma with SPECT and iodine-123-alpha-methyl tyrosine," Journal of Nuclear Medicine, vol. 39, no. 1, pp. 23-27, 1998.

[57] G. Arora, P. Sharma, A. Sharma et al., " $99 \mathrm{~m}$ Tc-Methionine hybrid SPECT/CT for detection of recurrent glioma:comparison with 18F-FDG PET/CT and contrast-enhanced MRI," Clinical Nuclear Medicine, vol. 43, no. 5, pp. e132-e138, 2018.

[58] H. Zhang, L. Ma, C. Wu, and B. N. Xu, "Performance of SPECT in the differential diagnosis of glioma recurrence from radiation necrosis," Journal of Clinical Neuroscience, vol. 22, no. 2, pp. 229-237, 2015.

[59] A. Amin, H. Moustafa, E. Ahmed, and M. El-Toukhy, "Glioma residual or recurrence versus radiation necrosis: accuracy of pentavalent technetium-99m-dimercaptosuccinic acid [Tc99m (V) DMSA] brain SPECT compared to proton magnetic resonance spectroscopy (1H-MRS): initial results," Journal of Neuro-Oncology, vol. 106, no. 3, pp. 579-587, 2011.

[60] N. Rani, B. Singh, N. Kumar et al., "Differentiation of recurrent/residual glioma from radiation necrosis using semi quantitative ${ }^{99 \mathrm{~m}}$ Tc MDM (Bis-Methionine-DTPA) brain SPECT/CT and dynamic susceptibility contrast-enhanced MR perfusion: a comparative study," Clinical Nuclear Medicine, vol. 43, no. 3, pp. e74-e81, 2018.

[61] A. Santra, P. Sharma, and R. Kumar, "Use of 99mtechnetium-glucoheptonate as a tracer for brain tumor imaging: an overview of its strengths and pitfalls," Indian Journal of Nuclear Medicine, vol. 30, no. 1, pp. 1-8, 2015. 


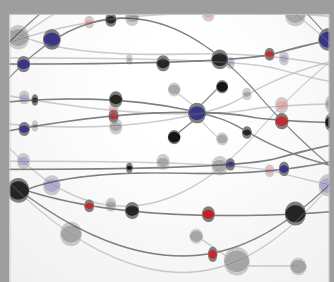

The Scientific World Journal
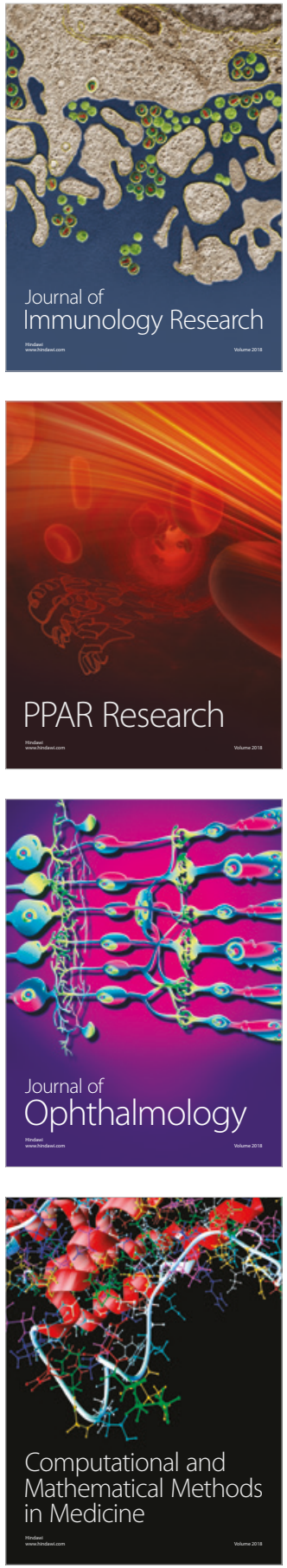

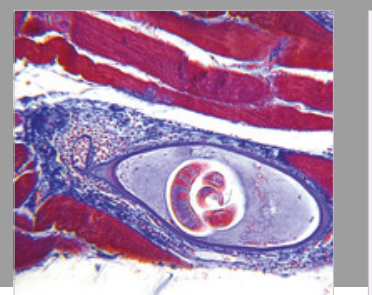

Gastroenterology Research and Practice

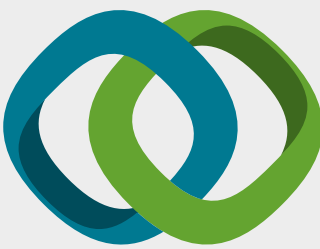

\section{Hindawi}

Submit your manuscripts at

www.hindawi.com
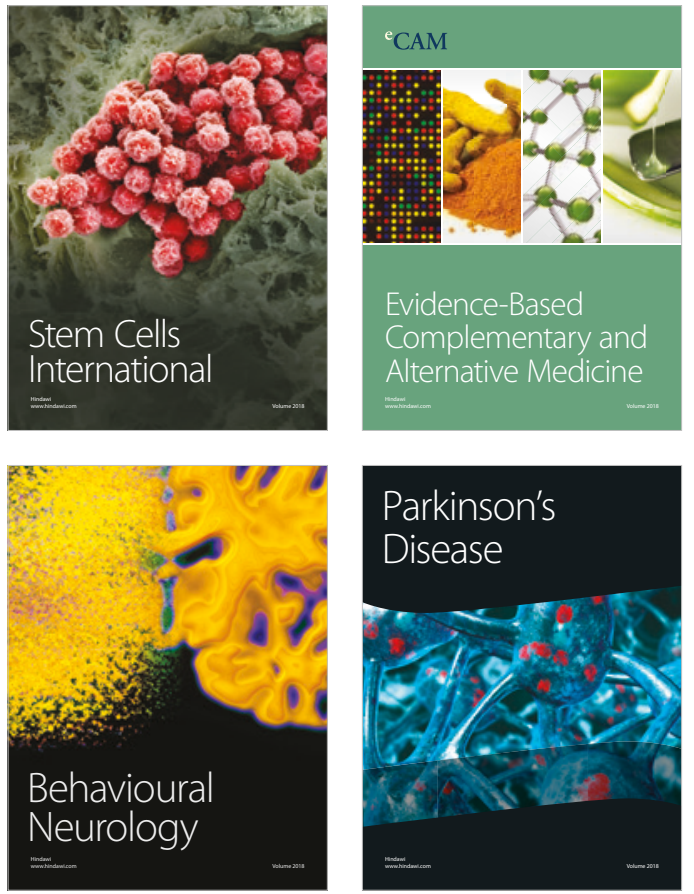

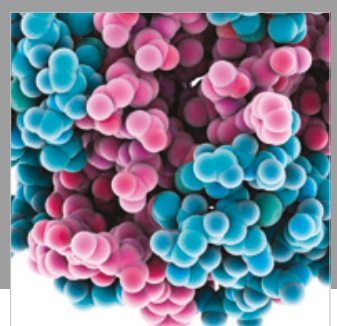

ournal of

Diabetes Research

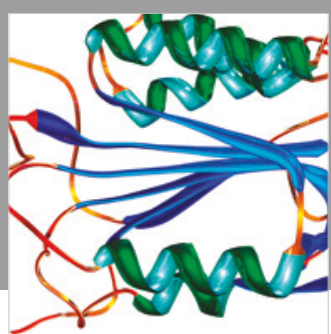

Disease Markers
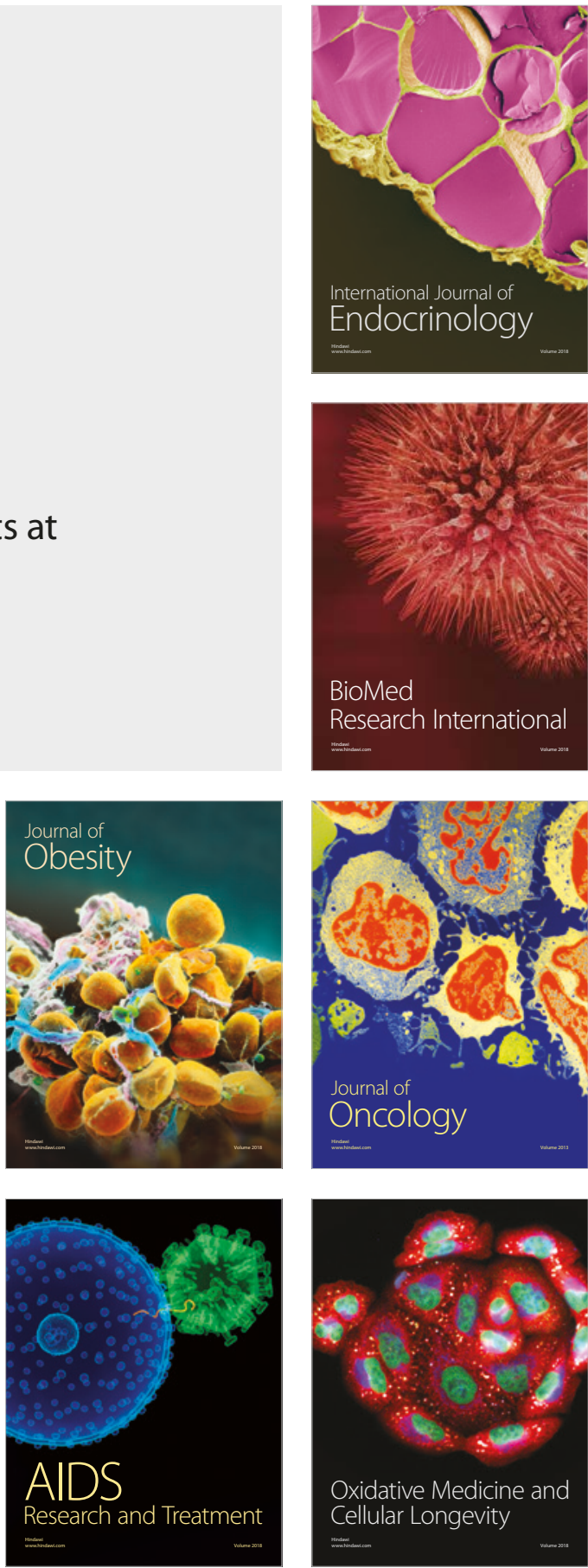